\title{
Comparison of the efficacy and safety of low-intensity extracorporeal shock wave therapy versus on-demand sildenafil for erectile dysfunction
}

\author{
Qi Lei, Dong Wang, Chunhui Liu, Zhigang Ji, Su Yan
}

Department of Urology, Peking Union Medical College Hospital, Beijing, China

Contributions: (I) Conception and design: All authors; (II) Administrative support: All authors; (III) Provision of study materials or patients: All authors; (IV) Collection and assembly of data: All authors; (V) Data analysis and interpretation: All authors; (VI) Manuscript writing: All authors; (VII) Final approval of manuscript: All authors.

Correspondence to: Su Yan, MD. Department of Urology, Peking Union Medical College Hospital, 1 Shuaifuyuan, Beijing 100730, China. Email: yansu@pumch.cn.

Background: Low-intensity extracorporeal shock wave therapy (Li-ESWT) is an effective therapy for
erectile dysfunction (ED) but is not widely recognized and applied. This prospective nonrandomized study
aimed to investigate the efficacy and safety of Li-ESWT.
Methods: After a 4-week washout period of past ED treatment, patients entered one of 2 active treatment
groups, either 9-week Li-ESWT or $100 \mathrm{mg}$ on-demand sildenafil. Patients were evaluated in the first- and
third-month following initiation of treatment. The Li-ESWT protocol comprised 2 sessions per week for
3 weeks, which were repeated after a 3 -week interval. Patients in the drug group took self-administered
sildenafil at a dose of $100 \mathrm{mg}$ before intercourse. The primary outcome was the effectiveness of Li-ESWT
measured by the International Index of Erectile Function-5 (IIEF-5) scores. Other measurements included
erection hardness score (EHS) and Self-Esteem And Relationship (SEAR).
Results: A total of 78 participants completed the study (46 in the Li-ESWT group and 32 in the sildenafil
group). Overall, $26.9 \%$ of the participants ( $21 / 78$ ) included were psychogenic. In the third month, the
outcome measured by IIEF-5 was 21.52 in the Li-ESWT group and 21.26 in the sildenafil group (P>0.05).
Proportion of improvement defined by minimal clinically important difference (MCID) criteria was $52.2 \%$
in the Li-ESWT group and $59.4 \%$ in the sildenafil group (P>0.05). The EHS and SEAR improvement
was similar in the 2 groups (P>0.05 at baseline and third month). Transient and mild adverse events were
observed in both groups.

Conclusions: In our study, a similar treatment efficacy and safety was shown by the application of LiESWT as on demand sildenafil.

Keywords: Erectile dysfunction (ED); low-intensity extracorporeal shock wave therapy; sildenafil; International Index of Erectile Function-5 (IIEF-5); treatment; psychogenic; organic

Submitted Jul 16, 2020. Accepted for publication Dec 02, 2020.

doi: $10.21037 /$ tau-20-1069

View this article at: http://dx.doi.org/10.21037/tau-20-1069

\section{Introduction}

Erectile dysfunction (ED) can be conceptualized as an impairment in the sexual response's arousal phase and is defined as the consistent inability to attain and/or maintain penile erection sufficient for sexual satisfaction, including satisfactory sexual performance $(1,2)$. It is a major male sexual disorder affecting $52 \%$ of men aged $40-70$ years, which is predicted to be a population of 322 million by 2025 worldwide. It is a multifactorial disease that influences the mental health and self-esteem of those affected and the 
satisfaction of sexual intercourse and quality of life for the couple.

Phosphodiesterase type 5 inhibitor (PDE5i) is considered a safe and effective therapy for ED, and works by relaxing smooth muscle cells and filling penile corpora cavernosa (3). However, the medication needs to be taken on-demand before sexual activity, which may be burdensome and inhibit sexual activity's spontaneity. Also, the medication needs to be taken long-term to maintain sufficient erectile function. Furthermore, for various reasons, $35 \%$ of patients may not respond to PDE5i (4).

Recently, low-intensity extracorporeal shockwave therapy (Li-ESWT), which was originally applied to ischemic heart disease, was introduced as a treatment for the penis (5). Erectile function is altered by Li-ESWT mainly through local neovascularization with multiple potential mechanisms, reversing pathologic processes in erectile tissue such as re-innervation, and reducing inflammation and oxidative stress (6). Several studies have shown the efficacy and safety of Li-ESWT for ED in both animal models $(7,8)$ and clinical trials $(4,5,8-18)$. Systematic reviews have also revealed that Li-ESWT could improve ED measured by both patient self-reports and instrument monitored outcomes $(17,19)$.

The efficacy and safety of PDE5i have been validated as a treatment for ED; Li-ESWT is also effective in treating ED, but has not yet been widely recognized. To our knowledge, there has been no comparative analysis of these 2 treatments using validated instruments. Herein, we designed a prospective nonrandomized interventional study to compare the efficacy and safety of on-demand 100 $\mathrm{mg}$ oral sildenafil and Li-ESWT therapy for ED patients. We present the following article in accordance with the TREND reporting checklist (available at http://dx.doi. org/10.21037/tau-20-1069).

\section{Methods}

We performed a prospective, nonrandomized, interventional study of 110 men who underwent initial screening, including medical history and physical examinations. One hundred men met the inclusion criteria; 60 patients chose Li-ESWT, and the other 40 chose sildenafil treatment. The study was conducted in accordance with the Declaration of Helsinki (as revised in 2013). The study was approved by the Ethics Committee of the Peking Union Medical College Hospital (\#S-K696) and informed consent was taken from all the patients.

\section{Enrollment inclusion and exclusion criteria}

Patients $\geq 18$ years of age, in a stable relationship, who reported $\geq$ a 3 -month history of ED and complained of insufficient erection to finish sexual intercourse were included. The diagnosis was made according to sexual and medical history, clinical examination, laboratory test results, and psychological evaluation. Patients were excluded on the grounds of anatomical abnormality, unstable medical (including clinically significant hepatobiliary or renal disease, and unstable cardiovascular disease) or psychiatric condition, a previous history of a neurological pathology, radical pelvic surgery, irradiations, or hormonal therapy, as well as ED caused by other sexual or endocrine disorders such as premature ejaculation or hypogonadism.

Participants of diverse pathogeneses were enrolled, including psychogenic, organic, and mixed ED, which was diagnosed with a medical history and psychological evaluation. Patients with positive findings, including diabetes, cardiovascular comorbidities, and negative nocturnal and morning erections, were diagnosed as organic or vascular pathogenesis. Men who had significant psychological evaluation changes with the presence of nocturnal or morning erections were diagnosed as psychogenic. Those who had both psychogenic and organic manifestations were diagnosed as mixed ED (20).

\section{Evaluation instruments}

At baseline, written informed consent and demographic data were provided by each participant. The study began with a screening period including assessment of erectile and sexual function, which was determined by several validated questionnaires widely used in clinical trials and clinical practice: International Index of Erectile Function-5 (IIEF-5) score, erection hardness score (EHS), and the impact of ED, which was determined using Self-Esteem And Relationship (SEAR) questionnaires consisting of Confidence Domain and Relationship Domain (17). The IIEF-5 score was intended for medical therapy evaluation. An EHS score of $\geq 3$ was defined as treatment success. The SEAR questionnaire was developed to indicate the negative effects of ED on the psychological condition and positive effects of successful treatment. Other indexes and parameters were obtained on admission, including patient demographics and other routine screening related to severe medical conditions. Participants also underwent clinical psychological assessment for anxiety and depression and nocturnal penile tumescence 


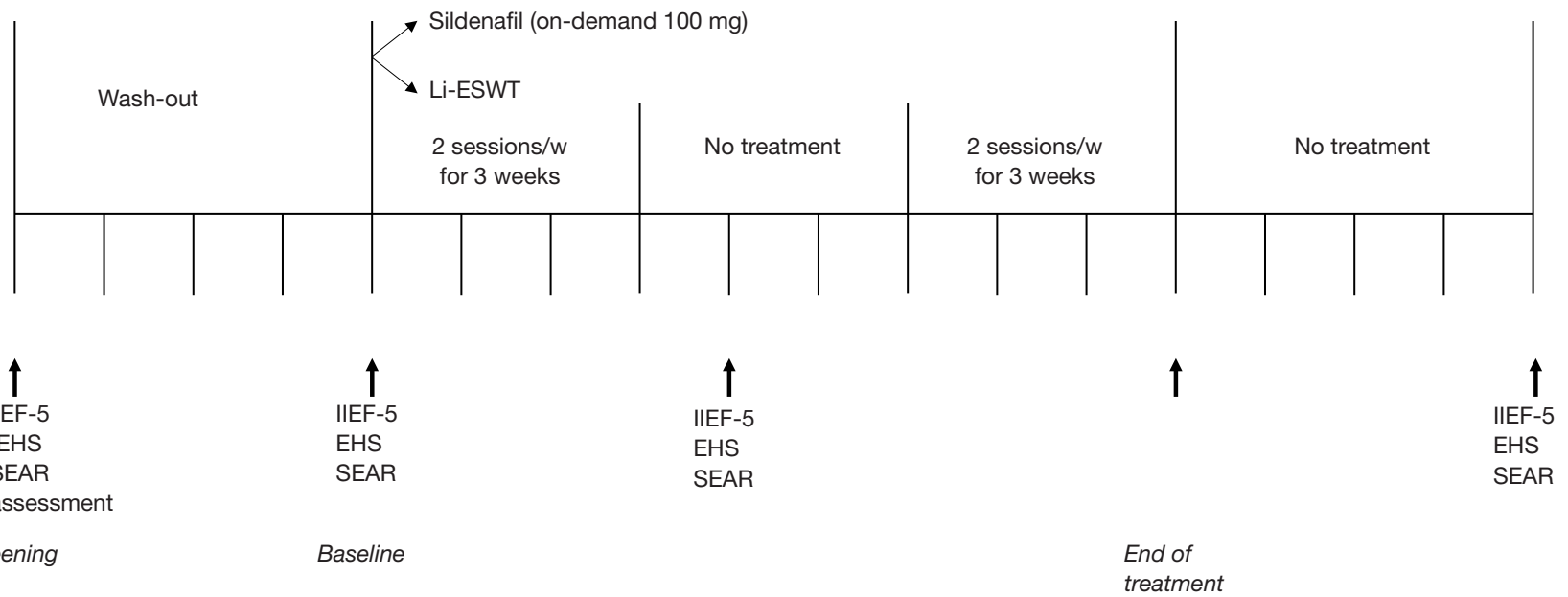

Figure 1 Study and treatment flow chart. IIEF-5, International Index of Erectile Function-5; EHS, Erectile Hardness Score; SEAR, SelfEsteem and Relationship Questionnaire; Li-ESWT, low-intensity extracorporeal shock wave therapy.

testing for 2 consecutive nights.

\section{Interventions}

After a 4-week washout period of past treatment and detailed explanation, participants entered one of 2 active treatment groups, either 9-week Li-ESWT or $100 \mathrm{mg}$ on-demand sildenafil therapy. The Li-ESWT protocol comprised of 2 sessions per week for 3 weeks, which were repeated after a 3-week interval, using Omnispec ED1000 (Medispec Ltd., Yehud, Israel) to produce low-intensity shockwaves (5). Li-ESWT was applied to each treatment session for $3 \mathrm{~min}$ at 5 different penile anatomical sites ( 3 locations on the penile shaft and 2 on the penile crura). Each Li-ESWT comprised 300 shocks per treatment point at an energy density of $0.09 \mathrm{~mJ} / \mathrm{mm}^{2}$ and a frequency of $120 /$ min. Participants in the Li-ESWT group were not permitted to take sildenafil or other kind of PDE5i pills for the trial duration. Participants in the drug treatment group self-administered sildenafil on-demand at a dose of $100 \mathrm{mg} 1$ hour before each event of intercourse. The IIEF5, EHS, and SEAR were assessed at the first and third months following the initiation of treatment (Figure 1). Side effect profile was assessed at every visit during the treatment period. The primary outcome was effectiveness as measured by IIEF-5 (21), with other measurements, including the EHS and SEAR. The number of adverse events assessed therapeutic safety. Participants of both groups were encouraged to engage in intercourse with their respective partners.

\section{Statistical analysis}

This study was a non-inferiority trial with the following null-hypothesis: Li-ESWT is non-inferior to sildenafil in terms of IIEF-5 score after 3-month of treatment. In a previous study, the mean IIEF-5 score after 3-month of treatment with sildenafil was $15.54 \pm 2.27$ (22). Therefore, for our sample size calculation, we set the non-inferiority margin of the difference between the 2 treatment groups to 1.5 (10\% of the mean IIEF-5 score after 3 -month of sildenafil treatment), and the standard deviation of 2.27. The ratio of participant numbers in the Li-ESWT and sildenafil groups were set at 2:1. Based on a two-group $t$-test of equivalence in means, using a one-sided significance level of $2.5 \%$, and a type II error of $20 \%$ (80\% power), this yielded a sample size of 81 patients (54 in the Li-ESWT group and 27 in the sildenafil group). To account for the estimated $10 \%$ of patients lost to follow-up or dropouts, at least 90 patients were required. Chi-Squared $\left(\chi^{2}\right)$ (or Fisher's exact) tests and independent sample $t$-tests were used to examine the distribution of key baseline characteristics for categorical and continuous variables, as well as to test the differences in dichotomous and continuous health parameters between both groups. Descriptive statistics for the duration of disease in both groups were shown as median along with the 25th (P25) and 75th percentile (P75) because of skewed distribution, and the Mann-Whitney $U$ test was used to compare the differences in median levels for the duration of disease between both groups. To determine the effect of the intervention on health parameters at the first- and 


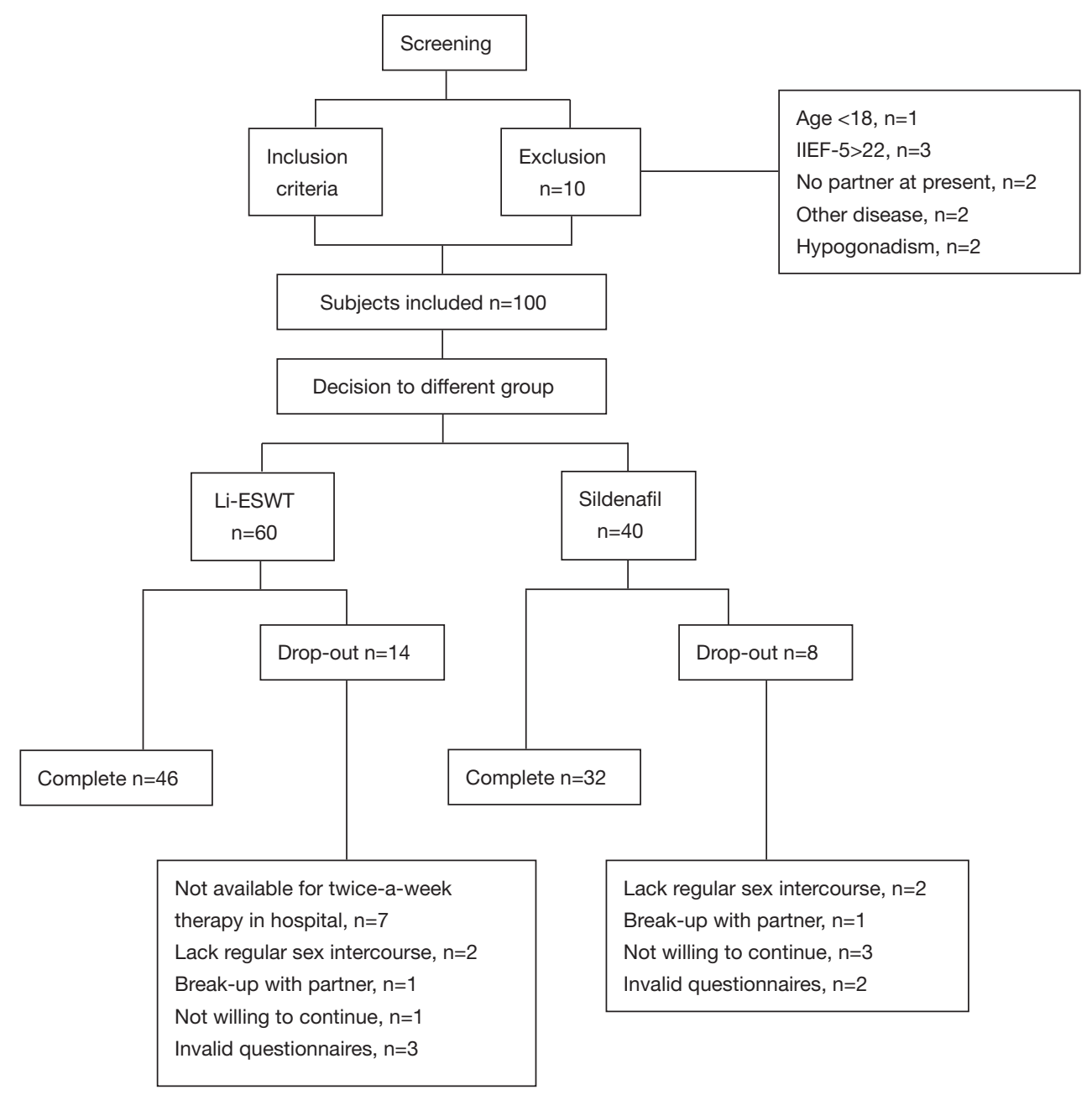

Figure 2 Screening, randomization. and follow-up flow chart. Li-ESWT, low-intensity extracorporeal shock wave therapy.

third-month following commencement of treatment, the estimated marginal mean in individuals of the 2 intervention groups was calculated using the SPSS general linear model (GLM) program (IBM, Armonk, NY, USA) adjusted for age, educational level, marriage status, and their baseline levels. A two-sided $\mathrm{P}$ value $<0.05$ was considered statistically significant. All data were analyzed using the statistical software SPSS version 20 (IBM Corp., Armonk, NY, USA).

\section{Results}

The first participant entered the study in April 2019, and the last completed in July 2019. Of 110 patients screened, 100 were enrolled (Figure 2). A total of 78 patients completed the study: the Li-ESWT group had 14 dropouts, and the sildenafil treatment group had 8 dropouts. The study was quit by 22 of 100 participants due to various reasons: unable to adhere to the treatment schedule $(7 / 100$, $7 \%)$, unable to have consistent regular sexual intercourse with their partner due to separation or break-up (6/100, $6 \%)$, and other reasons $(9 / 100,9 \%)$. These participants were not included in the data analysis. The included participants strictly followed the Li-ESWT protocol or reported sildenafil dosage during the trial. For 32 patients in the sildenafil group, the median dosage was 3.3 pills (100 mg sildenafil/pill) per week (1-6 pills) during the first month before the first follow-up, and 3.2 pills per week (1-6 pills) during the 2 months before the second followup. These patients' demographic profile was representative of the general population seen by urologists (Table 1). Treatment group demographics were similar at the baseline $(\mathrm{P}>0.05)$. 
Table 1 Baseline characteristics of participants in two treatment group

\begin{tabular}{|c|c|c|c|c|c|}
\hline \multirow{2}{*}{ Characteristics } & \multicolumn{2}{|c|}{ Li-ESWT $(n=46)$} & \multicolumn{2}{|c|}{ Sildenafil $(n=32)$} & \multirow{2}{*}{$P$ value } \\
\hline & $\mathrm{n}$ & $\%$ & $\mathrm{n}$ & $\%$ & \\
\hline Body mass index $\left[\mathrm{kg} / \mathrm{m}^{2}\right.$, mean (SD)] & \multicolumn{2}{|c|}{$24.3(4.0)$} & \multicolumn{2}{|c|}{$26.0(6.0)$} & $>0.05$ \\
\hline $\begin{array}{l}\text { Duration of disease [months, median } \\
\text { (P25-P75)] }\end{array}$ & \multicolumn{2}{|c|}{$24.0(12.0-36.0)$} & \multicolumn{2}{|c|}{$15.0(6.0-24.0)$} & $>0.05$ \\
\hline \multicolumn{5}{|l|}{ Severity } & \multirow[t]{3}{*}{$>0.05$} \\
\hline Mild to moderate & 23 & 50 & 19 & 59.4 & \\
\hline Moderate to severe & 23 & 50 & 13 & 40.6 & \\
\hline Elevated TC or TG & 0 & 0.0 & 2 & 6.2 & $>0.05$ \\
\hline Psychogenic & 12 & 26.1 & 9 & 28.1 & \multirow{3}{*}{$>0.05$} \\
\hline Organic & 17 & 37 & 11 & 34.3 & \\
\hline Mixed & 17 & 37 & 12 & 37.5 & \\
\hline \multicolumn{6}{|l|}{ Erectile function parameters } \\
\hline EHS & \multicolumn{2}{|c|}{$1.96 \pm 0.73$} & \multicolumn{2}{|c|}{$2.00 \pm 0.88$} & $>0.05$ \\
\hline IIEF-5 & \multicolumn{2}{|c|}{$14.09 \pm 3.75$} & \multicolumn{2}{|c|}{$13.00 \pm 4.20$} & $>0.05$ \\
\hline SEAR & \multicolumn{2}{|c|}{$35.89 \pm 9.48$} & \multicolumn{2}{|c|}{$36.25 \pm 12.08$} & $>0.05$ \\
\hline
\end{tabular}

ED, erectile dysfunction; Li-ESWT, Low-intensity extracorporeal shock wave therapy; TC, total cholesterol; TG, triglyceride; IIEF-5, International Index of Erectile Function-5; EHS, Erectile Hardness Score; SEAR, Self-Esteem and Relationship Questionnaire.

\section{Primary endpoint}

At baseline, the IIEF-5 score was equal in both groups $(14.09 \pm 3.75$ in the Li-ESWT group and $13.0 \pm 4.20$ in the sildenafil group, adjusted for age). The mean (SD) score in IIEF-5 for Li-ESWT and sildenafil was $19.0 \pm 5.75$ and $24.5 \pm 4.3$ at first month follow-up $(\mathrm{P}<0.01)$, and $20.52 \pm 5.92$ and $20.59 \pm 6.40$ at third month follow-up $(\mathrm{P}>0.05)$. Improvement of the IIEF-5 score was higher in the first month follow-up in the sildenafil group, with no statistically significant difference at the third month follow-up. The EHS and SEAR were similar to IIEF-5, which was equal at baseline, higher in the sildenafil group in the first month, but equal again in the third month (Table 2).

\section{Secondary endpoints}

Improvements were reported in $69.6 \%(32 / 46)$ of patients measured by IIEF-5 and $67.4 \%$ (31/46) by EHS and SEAR at the first month follow-up in the Li-ESWT group, and $96.8 \%(31 / 32)$ in the sildenafil group measured by IIEF$5,84.3 \%(27 / 32)$ by EHS and $90.6 \%(29 / 32)$ by SEAR (Table 2). At third month follow-up, the response ratio was higher than in the first month, with 78.3\% (36/46) in EHS, 80.4\% (37/46) in IIEF-5, and 71.7\% (33/46) in SEAR in the Li-ESWT group. Surprisingly, the response ratio was lower in the third month than the first month, with $81.3 \%(26 / 32)$ in IIEF-5, $78.1 \%(25 / 32)$ in EHS, and $62.5 \%(20 / 32)$ in SEAR in the sildenafil group.

According to minimal clinically important difference (MCID) criteria, a 7-score improvement of severe patients and 5 -score improvement of mild and moderate patients were recorded as positive results. In the third month, 24 participants (52.2\%) in the Li-ESWT group and 19 (59.4\%) in the sildenafil group reported positive results $(\mathrm{P}>0.05)$. In the third month, the ratio of patients who achieved clinical cure defined by IIEF-5 $>26$ was $21.9 \%$ in the sildenafil and $15.2 \%$ in the Li-ESWT group $\left(\kappa^{2}=0.57, \mathrm{P}>0.05\right)$. 
Table 2 The age-adjusted means of health parameters at the first- and third-month follow-ups after treatment by adjustment ${ }^{*}$

\begin{tabular}{|c|c|c|c|c|}
\hline Health parameters & Li-ESWT $(n=46)$, mean $(95 \% \mathrm{Cl})$ & Sildenafil $(n=32)$, mean $(95 \% \mathrm{Cl})$ & $\mathrm{F}$ & $\mathrm{P}$ \\
\hline EHS & $2.62(2.36-2.88)$ & 3.37 (3.09-3.66) & 16.35 & $<0.01$ \\
\hline IIEF-5 & $19.03(16.77-21.29)$ & $24.31(21.82-26.80)$ & 10.71 & $<0.01$ \\
\hline SEAR & 44.31 (39.62-48.99) & $54.14(48.99-59.29)$ & 8.67 & $<0.01$ \\
\hline EHS & $3.04(2.77-3.31)$ & $3.29(2.99-3.58)$ & 0.75 & $>0.05$ \\
\hline IIEF-5 & $21.52(18.90-24.14)$ & $21.26(18.38-24.14)$ & 0.02 & $>0.05$ \\
\hline SEAR & $48.36(42.75-53.96)$ & $47.94(41.77-54.11)$ & 0.01 & $>0.05$ \\
\hline
\end{tabular}

*, means were adjusted for age by GLM model. Li-ESWT, low-intensity extracorporeal shock wave therapy; Cl, confidence interval; IIEF-5, International Index of Erectile Function-5; EHS, Erectile Hardness Score; SEAR, Self-Esteem and Relationship Questionnaire.

Table 3 Adverse events in the 2 groups

\begin{tabular}{|c|c|c|c|c|}
\hline Adverse event & \multicolumn{2}{|c|}{ Li-ESWT } & \multicolumn{2}{|c|}{ Sildenafil } \\
\hline Flush & 3 & 9.4 & 0 & \\
\hline Headache and dizziness & 1 & 3.1 & 1 & 2.2 \\
\hline Dyspepsia & 1 & 3.1 & 1 & 2.2 \\
\hline Others & 0 & & 0 & \\
\hline
\end{tabular}

Li-ESWT, low-intensity extracorporeal shock wave therapy.

\section{Safety}

All enrolled participants were included in the safety evaluation. There was no participant discontinuation due to adverse events. The most common treatment-emergent adverse events in the sildenafil group were flushing, headache and dizziness, and dyspepsia (Table 3). The most common treatment-emergent adverse events in the LiESWT group were headache and dizziness, dyspepsia, and local penile pain.

\section{Discussion}

According to our study and analysis, Li-ESWT showed similar efficacy for general ED patients as sildenafil, with minor safety concerns.

Oral therapy of on-demand sildenafil is effective in the treatment of ED of diverse pathological origins and with various comorbidities (20). In recent studies, attempts have been made to establish the safety and efficacy of Li-ESWT in ED (4,5,8-18). More evidence is needed to support the use of Li-ESWT for ED in clinical practice (23). In this study, we have directly compared these 2 therapies for ED treatment.

We observed that Li-ESWT showed a similar treatment outcome to sildenafil as measured by IIEF-5 and other instruments in general ED patients. At the third month follow-up, similar outcomes were observed in both groups as measured by IIEF-5, EHS, and SEAR after commencing treatment. At first month follow-up, parameters were higher in the sildenafil group, indicating immediate improvement after medication. A longer duration may be required by $\mathrm{Li}-$ ESWT to show its efficacy, which, on the other hand, was sustained over a longer period. Besides, patients were still undergoing the second 3-week Li-ESWT therapy at the first month follow-up assessment, and theoretical benefit from Li-ESWT might not have been achieved by then. The improvement proportion of patients defined by MCID 
criteria showed similar treatment effects. The improvement proportion of SEAR and EHS also had no statistically significant difference in both groups.

The improvements of IIEF-5 and EHS measurements indicated both therapies were effective for improving erectile function. Elevation of the SEAR score also indicated that regular and voluntary treatment could improve the psychological condition, especially regarding SEAR with partner.

More time was required for Li-ESWT to achieve its therapeutic effect. In secondary endpoints, we observed continuous improvements in the Li-ESWT group at third-month follow-up compared with the first month, which was especially reflected by the SEAR score. Rather than taking pills just before sexual intercourse, Li-ESWT provided a new treatment pattern that was more stable and proactive, helping participants to reestablish their confidence. This novel therapeutic method has fulfilled part of ED patients' unmet need, including those who could not take oral medication due to clinical concerns or subjective rejection (24).

Our results indicated that Li-ESWT was another alternative to PDE5i for ED patients. Compared to sildenafil, Li-ESWT has shown more potential to achieve a long-term therapeutic effect due to its addressing underlying neovascularization mechanisms (25), which is advantageous to this novel therapy. Sildenafil is dose-dependent, and clinical dose adjustments depend on the condition of each individual. The maximum dose of sildenafil was used in our study to achieve a significant therapeutic effect.

We included ED patients of organic, psychogenic, and mixed origins, general ED patients treated with sildenafil. Improvement of erectile hardness could also significantly elevate confidence during sexual intercourse (26). Thus, it is reasonable to expect Li-ESWT also to be effective for psychogenic ED (27). This study showed that organic, psychogenic, and mixed origin participants all improved in IIEF-5 and EHS with statistical significance. Improvements in the SEAR score further substantiated this evidence.

Both therapies were shown to be safe for participants included in this study. None of them quit the study due to adverse events, despite various adverse events observed in both groups. In the sildenafil group, $9.4 \%$ of patients experienced transient flush at least once. Headache, dizziness, and dyspepsia were also observed in both the sildenafil group and the Li-ESWT group. One participant in the Li-ESWT group reported transient penile local pain.

This prospective nonrandomized interventional comparison study recapitulated clinical practice compared with a perfectly designed placebo-controlled trial. Sildenafil is a well-known therapy in clinical practice, while LiESWT treatment is novel. All patients included in the study received free guidance and regular visits, which elevated the participant compliance.

However, participants' treatment outcomes were multifactorial and determined by more than therapy. Other factors such as severity and duration of $\mathrm{ED}$, comorbidities, lifestyle, and relationship influenced the compliance and the outcome of treatment. Successful sexual intercourse is a collaborative act between partners. Also, patients' heavy work may contribute to an unhealthy lifestyle, which consequently results in unsuccessful or unsustainable erections.

Our study had several limitations. First, our study was single centered, with most patients from 1 hospital, which might have introduced a participant selection. The participants in our study were relatively young (mean age 33.4 in Li-ESWT and 30.7 in sildenafil). They were more likely to be affected by honeymoon impotence, and may not have accurately represented a general ED population; the enrolled participants were more likely to quit ED treatment. Second, the number of participants was not large enough for subgroup comparison concerning psychogenic, vascular, and mixed causes. The participants experiencing ED from different causative factors might have had different responses and improvements to each therapy. Finally, the participants elected to enter either the Li-ESWT or sildenafil group after receiving a detailed explanation of both therapies. As a result of this nonrandomization, participants were older overall in the $\mathrm{Li}$ ESWT group. Analyses were performed after adjusting for age using the GLM model. Besides, baseline characteristics suggested no significant difference between the 2 groups of patients. However, our study investigated Li-ESWT in a Chinese population with large enrolments using validated questionnaires. And the prospective design helped eliminate information collection bias. We also performed comprehensive evaluation of the enrolled participants, which provided a more detailed description of patients' baseline situation.

\section{Conclusions}

Our study showed that Li-ESWT had similar efficacy as on-demand sildenafil for general ED patients, as measured by validated instruments. Therefore, Li-ESWT provides 
another option for patients experiencing ED. However, future studies are needed to explore modifications for therapeutic schemes and the improvement of parameters.

\section{Acknowledgments}

We would like to acknowledge all staff in the Department of Urology of PUMCH for assisting in this program, Medispec Inc., and Beijing Weili New Century Science and Tech. Deve. Co. Ltd. for provision of the therapeutic instruments.

Funding: None.

\section{Footnote}

Reporting Checklist: The authors have completed the TREND reporting checklist. Available at http://dx.doi. org/10.21037/tau-20-1069

Peer Review File: Available at http://dx.doi.org/10.21037/ tau-20-1069

Data Sharing Statement: Available at http://dx.doi. org/10.21037/tau-20-1069

Conflicts of Interest: All authors have completed the ICMJE uniform disclosure form (available at http://dx.doi. org/10.21037/tau-20-1069). The authors have no conflicts of interest to declare.

Ethical Statement: The authors are accountable for all aspects of the work in ensuring that questions related to the accuracy or integrity of any part of the work are appropriately investigated and resolved. The study was conducted in accordance with the Declaration of Helsinki (as revised in 2013). The study was approved by the Ethics Committee of the Peking Union Medical College Hospital (\#S-K696) and informed consent was taken from all the patients.

Open Access Statement: This is an Open Access article distributed in accordance with the Creative Commons Attribution-NonCommercial-NoDerivs 4.0 International License (CC BY-NC-ND 4.0), which permits the noncommercial replication and distribution of the article with the strict proviso that no changes or edits are made and the original work is properly cited (including links to both the formal publication through the relevant DOI and the license).
See: https://creativecommons.org/licenses/by-nc-nd/4.0/.

\section{References}

1. NIH. Consensus development conference statement. National Institutes of Health. Impotence. December 7-9, 1992. Int J Impot Res 1993;5:181-284.

2. Burnett AL, Nehra A, Breau RH, et al. Erectile Dysfunction: AUA Guideline. J Urol 2018;200:633-41.

3. Konstantinos G, Petros P. Phosphodiesterase-5 inhibitors: future perspectives. Curr Pharm Des 2009;15:3540-51.

4. Gruenwald I, Appel B, Vardi Y. Low-intensity extracorporeal shock wave therapy--a novel effective treatment for erectile dysfunction in severe ED patients who respond poorly to PDE5 inhibitor therapy. J Sex Med 2012;9:259-64.

5. Vardi Y, Appel B, Jacob G, et al. Can low-intensity extracorporeal shockwave therapy improve erectile function? A 6-month follow-up pilot study in patients with organic erectile dysfunction. Eur Urol 2010;58:243-8.

6. Sokolakis I, Dimitriadis F, Teo P, et al. The Basic Science Behind Low-Intensity Extracorporeal Shockwave Therapy for Erectile Dysfunction: A Systematic Scoping Review of Pre-Clinical Studies. J Sex Med 2019;16:168-94.

7. Qiu X, Lin G, Xin Z, et al. Effects of low-energy shockwave therapy on the erectile function and tissue of a diabetic rat model. J Sex Med 2013;10:738-46.

8. Assaly-Kaddoum R, Giuliano F, Laurin M, et al. Low Intensity Extracorporeal Shock Wave Therapy Improves Erectile Function in a Model of Type II Diabetes Independently of NO/cGMP Pathway. J Urol 2016;196:950-6.

9. Vardi Y, Appel B, Kilchevsky A, et al. Does low intensity extracorporeal shock wave therapy have a physiological effect on erectile function? Short-term results of a randomized, double-blind, sham controlled study. J Urol 2012;187:1769-75.

10. Gruenwald I, Kitrey ND, Appel B, et al. Low-Intensity Extracorporeal Shock Wave Therapy in Vascular Disease and Erectile Dysfunction: Theory and Outcomes. Sex Med Rev 2013;1:83-90.

11. Vardi Y, Appel B, Kitrey ND, et al. 604 Additional shockwave treatment improves erectile function in patients with poor response to the standard shockwave protocol. Eur Urol Suppl 2014;13:e604.

12. Vardi Y, Appel B, Kitrey ND, et al. 603 Low-intensity shockwave treatment for ED - long term follow up of 2 years. Eur Urol Suppl 2014;13:e603. 
13. Reisman Y, Hind A, Varaneckas A, et al. Efficacy and safety of linear focused shockwaves for erectile dysfunction (renova) - a second generation technology. J Sex Med 2014;11:243.

14. Iacono F, Ruffo A, Prezioso D, et al. Low intensity shock wave (LISW) treatment (renova) in order to improve male sexual function: a preliminary data on 42 patients. J Sex Med 2014;11:24.

15. Kitrey ND, Gruenwald I, Appel B, et al. Penile Low Intensity Shock Wave Treatment is Able to Shift PDE5i Nonresponders to Responders: A Double-Blind, Sham Controlled Study. J Urol 2016;195:1550-5.

16. Kalyvianakis D, Hatzichristou D. Low-Intensity Shockwave Therapy Improves Hemodynamic Parameters in Patients With Vasculogenic Erectile Dysfunction: A Triplex Ultrasonography-Based Sham-Controlled Trial. J Sex Med 2017;14:891-7.

17. Sokolakis I, Hatzichristodoulou G. Clinical studies on low intensity extracorporeal shockwave therapy for erectile dysfunction: a systematic review and metaanalysis of randomised controlled trials. Int J Impot Res 2019;31:177-94.

18. Patel P, Huang C, Molina M, et al. Clinical trial update on shockwave therapy and future of erectile function restoration. Int J Impot Res 2019;31:206-8.

19. Dong L, Chang D, Zhang X, et al. Effect of Low-Intensity Extracorporeal Shock Wave on the Treatment of Erectile
Dysfunction: A Systematic Review and Meta-Analysis. Am J Mens Health 2019;13:1557988319846749.

20. Burnett AL, Nehra A, Breau RH, et al. Erectile Dysfunction: AUA Guideline. J Urol 2018;200:633-41.

21. Rosen RC, Allen KR, Ni X, et al. Minimal clinically important differences in the erectile function domain of the International Index of Erectile Function scale. Eur Urol 2011;60:1010-6.

22. Wang R, Zheng T, Zhang TB, et al. Efficacy and safety of regular use of sildenafil in the treatment of penile erectile dysfunction. Zhonghua Nan Ke Xue 2019;25:603-7.

23. Fode M, Albersen M, Ostergren PB. Is low-intensity shockwave therapy for erectile dysfunction ready for clinical practice? Int J Impot Res 2019;31:204-5.

24. Yang H, Seftel AD. Controversies in low intensity extracorporeal shockwave therapy for erectile dysfunction. Int J Impot Res 2019;31:239-42.

25. Gruenwald I, Albersen M. Editors Summary: The future of shockwave therapy. Int J Impot Res 2019;31:243.

26. Sontag A, Ni X, Althof SE, et al. Relationship between erectile function and sexual self-confidence: a path analytic model in men being treated with tadalafil. Int J Impot Res 2014;26:7-12.

27. Liu T, Shindel AW, Lin G, et al. Cellular signaling pathways modulated by low-intensity extracorporeal shock wave therapy. Int J Impot Res 2019;31:170-6.
Cite this article as: Lei Q, Wang D, Liu C, Ji Z, Yan S. Comparison of the efficacy and safety of low-intensity extracorporeal shock wave therapy versus on-demand sildenafil for erectile dysfunction: a prospective non-randomized study. Transl Androl Urol 2021;10(2):860-868. doi: 10.21037/tau-201069 